\title{
Silver/Zinc oxide self-assembled nanostructured
}

\section{bolometer}

John E. Sanchez ${ }^{1}$, Gabriel Gonzáleż, Gustavo Vera-Reveles ${ }^{3}$, J. Jesus Velazquez-Salazar ${ }^{1}$, Lourdes Bazan-Diaz ${ }^{1}$, José M. Gutiérrez-Hernández ${ }^{3}$ Miguel José-Yacaman ${ }^{1}$, Arturo Ponce ${ }^{1}$, and Francisco J. González **

${ }^{1}$ Department of Physics and Astronomy, University of Texas at San Antonio, San Antonio 78249, $U S A$.

${ }^{2}$ Cátedras CONACYT, Universidad Autónoma de San Luis Potosí, San Luis Potosí, 78000, MEXICO.

${ }^{3}$ Coordinación para la Innovación y la Aplicación de la Ciencia y la Tecnología, Universidad Autónoma de San Luis Potosí, San Luis Potosí, 78000, MEXICO.

*Corresponding author: javier.gonzalez@uaslp.mx

KEYWORDS: Infrared detectors, microbolometers, self-assembled, zinc oxide nanostructures. 


\begin{abstract}
Temperature coefficient of resistance (TCR) is the main figure of merit for bolometric detectors. Reports show that zinc oxide can have TCR values higher than vanadium oxide $\left(\mathrm{VO}_{\mathrm{x}}\right)$ and amorphous silicon (a-Si), which are the most common materials used in bolometric applications, however its high resistivity makes it difficult to match it to read-out electronics. In this work, self-assembled nanostructures of Silver/Zinc oxide $(\mathrm{Ag} / \mathrm{ZnO})$ have been fabricated as well as their electrical and optical properties were measured as function of the $\mathrm{Ag} / \mathrm{ZnO}$ concentration ratio. It was found that the nanostructures with the highest $\mathrm{ZnO}$ concentration exhibited a temperature coefficient of resistance as high as $-11.8 \% \mathrm{~K}^{-1}$ near room temperature. Moreover, the TCR values and conductivity of the material can be tuned with the Ag/ $\mathrm{ZnO}$ concentration ratio. This tuning flexibility allows this material to be better matched to read-out integrated circuits.
\end{abstract}




\section{Introduction}

Bolometric detectors are key components in uncooled infrared imaging systems, their main figure of merit, which determines their responsivity, is the temperature coefficient of resistance (TCR). The TCR is defined as the percentage change in resistance as a function of temperature, if the resistance increases with an increase in temperature it is assigned a positive coefficient of resistance for that material. However, if the inverse occurs, the material will exhibit a negative coefficient, which means the resistance decreases as temperature increases [1]. The TCR values of materials used for bolometric detectors, such as vanadium oxide $\left(\mathrm{VO}_{\mathrm{x}}\right)$ or amorphous silicon (a-Si), varies around 2-3\% $\mathrm{K}^{-1}[1]$. Bolometric detectors work by presenting a change in resistance due to an increase in temperature which is a result of the absorption of incident electromagnetic radiation, this change in resistance can be translated to a change in voltage if the device is biased by an electrical current, in this case a figure of merit for bolometric detectors would be the voltage responsivity $R_{V}$, which is the output signal voltage per incident electromagnetic power, given by [1]:

$$
R_{V}=\frac{I_{b} R \alpha \eta}{G \sqrt{1+\omega^{2} \tau^{2}}}
$$

where $I_{b}$ is the bias current, $R$ is the dc resistance, $\alpha$ is the temperature coefficient of resistance (TCR), $\eta$ is the absorptivity, $G$ is the thermal conductance between the sensitive element and the substrate, $\omega$ is the angular modulation frequency of the incident radiation, and $\tau$ is the thermal time constant. Furthermore, it is important to point another important parameter for bolometric detectors, the noise equivalent power (NEP), which is the input power level that will produce a 
signal-to-noise ratio (SNR) of 1, the units of NEP are watts and it can be written in terms of responsivity as:

$$
N E P=\frac{V_{n}}{R_{V}} .
$$

Therefore, low-noise devices with high TCR would make good bolometric detectors. Current infrared imaging systems use $\mathrm{VO}_{\mathrm{x}}$ as the bolometric material, which has a TCR around $-3 \% \mathrm{~K}^{-1}$. In order to increase the responsivity of current infrared imaging systems a great effort has been put towards finding alternatives to currently standard bolometric materials [2-4].

Recently, zinc oxide $(\mathrm{ZnO})$ has attracted a lot of attention due to its TCR values, which have been reported in the range from $-3.4 \% \mathrm{~K}^{-1}$ to $-10.4 \% \mathrm{~K}^{-1}$ at room temperature [5]. These values are higher than the ones for $\mathrm{VO}_{\mathrm{x}}$ and $\mathrm{a}-\mathrm{Si}$, which are the most common materials used in infrared imaging systems [1]. The main problem with $\mathrm{ZnO}$ is its high resistivity which translates into high noise and a poor impedance match to read-out integrated circuits [1]. Previous work has shown that an increase in TCR can be obtained by using nanostructured materials, Wang et al. reported a TCR of $-6.5 \% \mathrm{~K}^{-1}$ for nanostructured thin films of vanadium oxide [6], and Choi et al. reported a TCR of $-7.06 \% \mathrm{~K}^{-1}$ for $\mathrm{VO}_{2}$ nanowires at $300 \mathrm{~K}$ [7]. Also doping of $\mathrm{ZnO}$ has been used to increase the TCR of $\mathrm{ZnO}$, Zhou reported a TCR value of $-17.5 \% \mathrm{~K}^{-1}$ at $244 \mathrm{~K}$ for bolometers based on $\mathrm{ZnO}$ films doped with silver [8]. Furthermore, the possibility of having a mixture of intermetallic-semiconductor nanostructures makes it feasible to tailor the resistance of the active component on the device in order to match it to read-out integrated circuits and reduce the electrical noise of the device [9]. 
In this article we investigate the bolometric characteristics of self-assembled metalsemiconductor nanostructures made of silver nanowires and zinc oxide nanorods in a hierarchical arrangement. To show the efficiency of the bolometric nanomaterial, measurements of TCR, absorption and electrical noise of the $\mathrm{Ag} / \mathrm{ZnO}$ nanostructures are presented as a function of the $\mathrm{Ag} / \mathrm{ZnO}$ concentration ratio.

\section{Fabrication and nanostructure characterization}

The procedure for the fabrication of the $\mathrm{Ag} / \mathrm{ZnO}$ nanostructure bolometer has been outlined previously using a chemical microwave method [10]. Briefly, the Ag/ZnO metal-semiconductor nanostructures are synthesized by establishing a two steps process. First, silver nanowires (AgNWs) were fabricated by following the polyol method: $5 \mathrm{ml}$ of ethylene glycol (EG) were heated at $160^{\circ} \mathrm{C}$ for 40 min.; next, a silver nitrate $\mathrm{AgNO}_{3}$ (reagent grade $99.99 \%$ by Sigma- Aldrich) is reduced in a solution of EG following the addition of polyvinyl pyrrolidone (PVP, Mw 55,000 reagent grade $99.99 \%$ by Sigma-Aldrich); (EG) and (PVP) act as reducer solution and capping agent to polar molecules, respectively. The mixture is subjected to a constant stirring rate for a period time, 40 to 60 min, until the silver nanowires reach the desired and most stable diameter ( $\sim 70 \mathrm{~nm}$ ) and length (more than $2 \mu \mathrm{m}$ ), as described in Refs. [11] and [12].

As for the second part, during the self- assembling process of $\mathrm{ZnO}$ nanorods on silver nanowires (Ag-NWs), Zinc acetate dehydrate (Zn(Ac)2, 98\% reagent by Sigma-Aldrich), 51725 mM and hexamethylenetetramine (HMT) $51725 \mathrm{mM}$ were dissolved in deionized water to form a precursor initial solution; next, $200 \mu \mathrm{l}$ of silver nanowires, as obtained by the polyol method, were added to the former solution, which is now irradiated using an ETHOS EZ Microwave Digestion System, working within 400 to $700 \mathrm{~W}$ at a microwave frequency of $2.5 \mathrm{GHz}$. The vial containing the precursor solution is heated between $20^{\circ} \mathrm{C}-90^{\circ} \mathrm{C}$, with an exposure reaction time 
from 1 to 50 min. Figure 1 (a) shows a typical $\mathrm{ZnO} / \mathrm{Ag}$ nanostructure, $\mathrm{ZnO}$ nanorods grow from the pentagonal sides of the Ag nanowire producing a five element star-shaped structure (Figure 1 (b)).

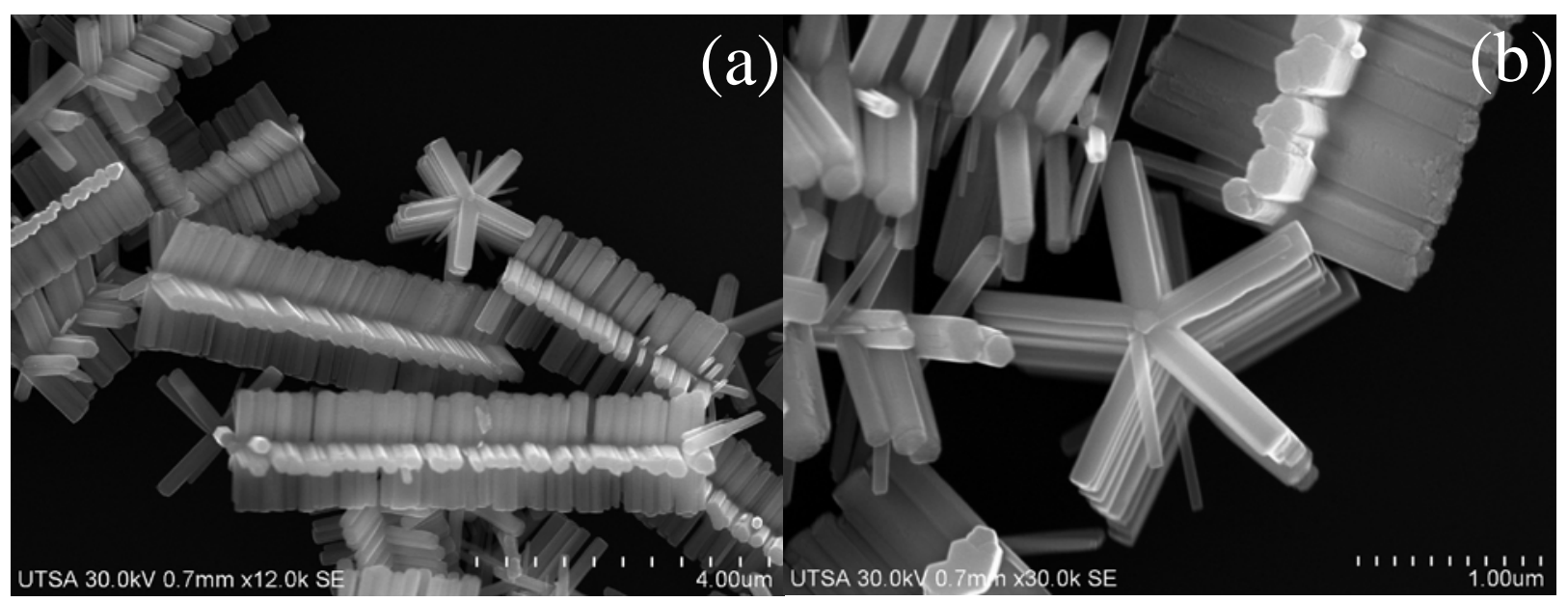

Figure 1. Multi-pentagonal arrangement of zinc oxide nanorods growing from the pentagonal faces on silver nanowires. (a) SEM images shows the normalized distribution of $\mathrm{ZnO}$ nanorods along Ag Nws. (b) High magnification image of the multi-segmented arrangement.

The $\mathrm{ZnO}$ nanorods are assembled in the lateral surfaces of the silver nanowires, which have a pentagonal cross section area. In this way, there are five nucleation sites for the $\mathrm{ZnO}$ nanorods which are oriented along the [001] direction on the (110) planes of the silver. The analysis of the crystalline orientation has been performed by using precession electron diffraction-assisted automated crystal orientation mapping (PED ACOM-TEM) [13]. PED ACOM-TEM gives the full crystallographic information of the object at a specific area of the region of interest and provides a crystallographic map at the nanometer scale. In the ACOM-TEM technique the electron beam is scanned across the sample and collects the electron diffraction patterns using a CCD camera attached to the viewing screen of the 
microscope. All of the electron diffraction patterns collected are then indexed using an automatic matching procedure, identified by a cross-correlation with preselected theoretical templates from a database. In Fig. 2 a crystal orientation map is shown. Figures 2(a), 2(b) and 2(c) correspond to the orientation map in the directions $x, y$ and $z$, respectively. Figures 2(d) and 2(e) show a scanning electron microscopy (SEM) image and a cartoon of the assembled hierarchical nanostructure $\mathrm{ZnO} / \mathrm{Ag}$. Below figures 2(d) and 2(e) the color chart extracted from the pole figures is shown. The color code indicates the orientation near to the $<110>$ zone axis (green color) for the orientation in $\mathrm{x}$ in silver, which correspond with the parallel direction of the electrons path within the column of the transmission electron microscope. $\mathrm{ZnO}$ nanorods show distinct orientations due to the fact that the hexagonal rods are rotated in perpendicular directions of the silver nanowires and along their [001] defections. 


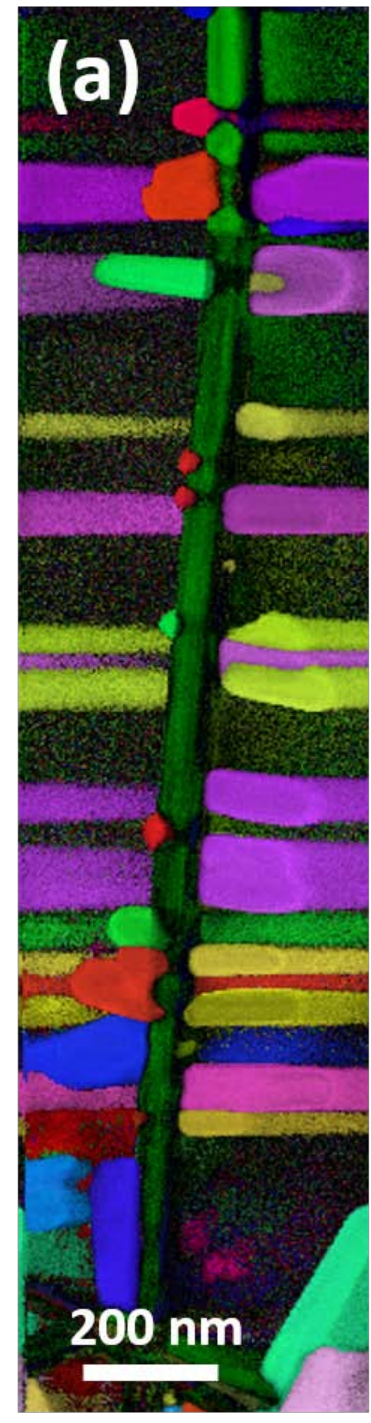

Orientation $x$

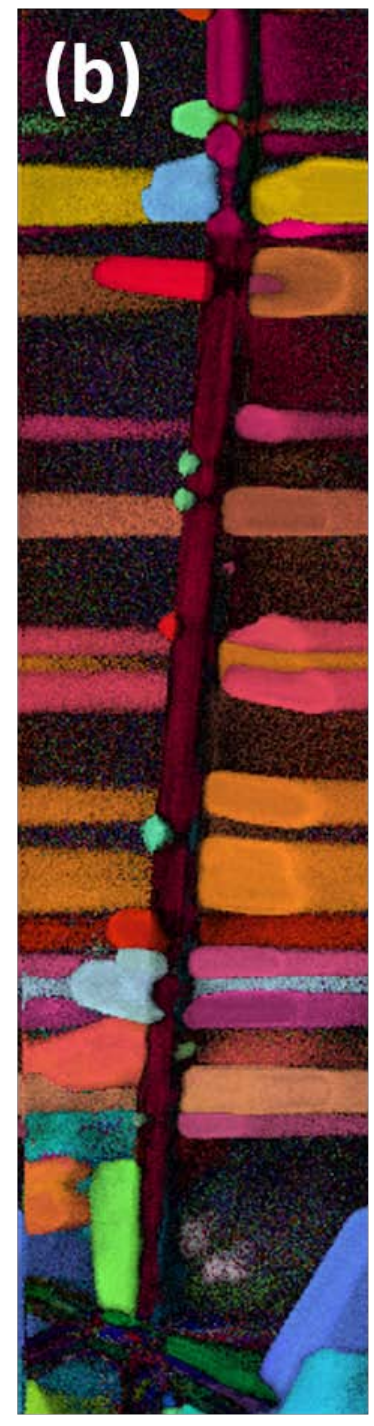

Orientation $y$

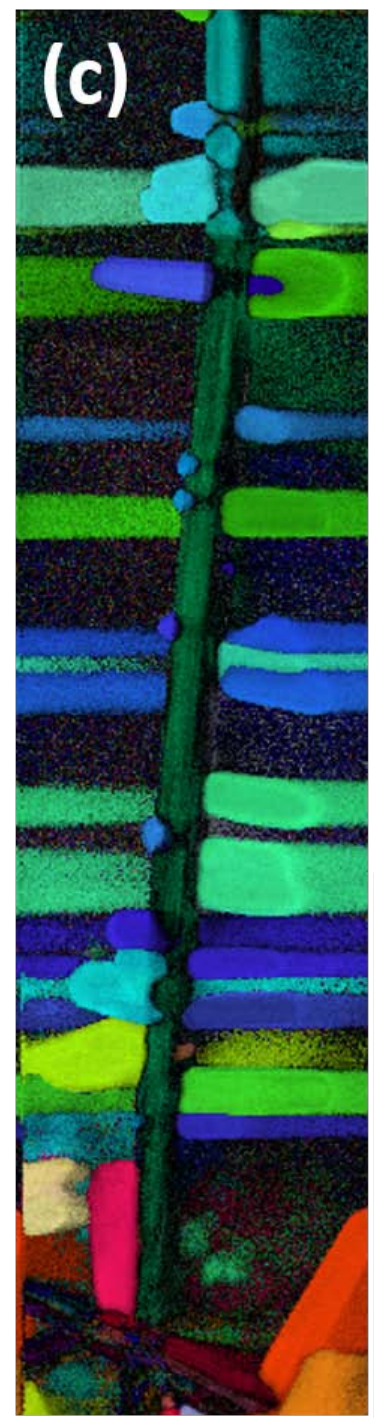

Orientation $z$
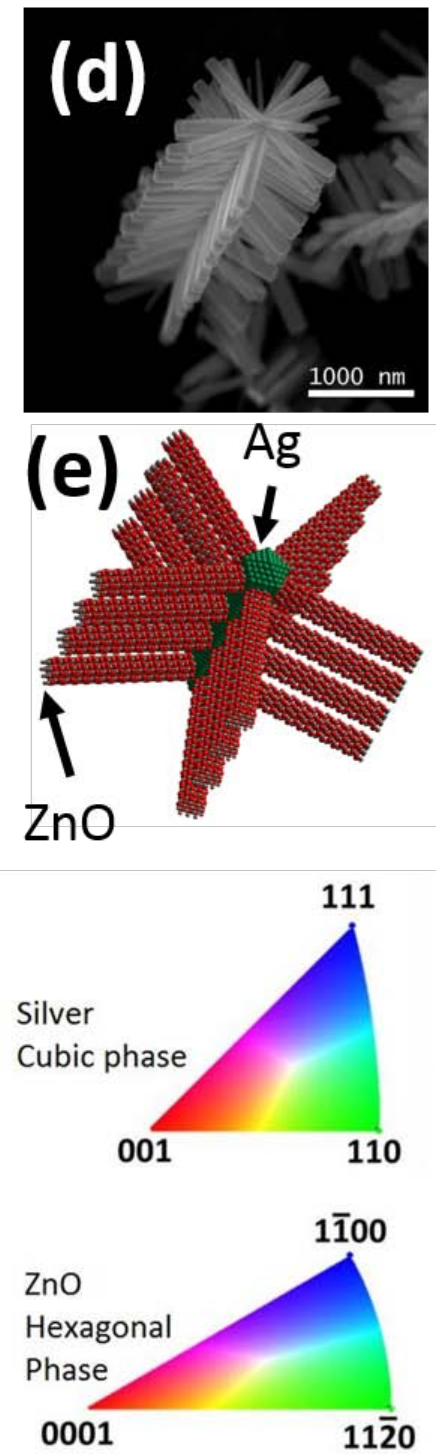

Figure 2. Crystalline orientation maps using PED ACOM-TEM: orientations (a) $\mathrm{x}$, (b) y and

(c) z. (d) SEM micrograph of the ZnO/Ag assembling and (e) graphical representation of the epitaxial growing.

The solutions were then deposited on Si substrates by drop coating and then were put to dry, silver paint was used to make electrodes on the bolometric films in order to make the electrothermal measurements. It is worth noting that no degradation over time was observed with the 
bolometric performance of the dried nanostructured solutions, the same bolometric characteristics have been obtained in samples that were at least one year old.

Three set of samples with different silver-zinc oxide ratios were prepared, the first sample consisted of pure silver nanowires (Fig. 3(a)), the second sample was made from Ag/ZnO nanostructures fabricated using the standard procedure presented (Figures 1(a) and 1(b)) and the third set of samples was obtained by introducing $\mathrm{ZnO}$ nanoparticles as seeds to increase the $\mathrm{ZnO}$ content (Figures 3(c) and 3(d)). By using ZnO nanoparticles as seeds an increased number of $\mathrm{ZnO}$ nanorods grow on the $\mathrm{Ag} / \mathrm{ZnO}$ nanostructures increasing the $\mathrm{ZnO}$ concentration (Fig. 3(d)).

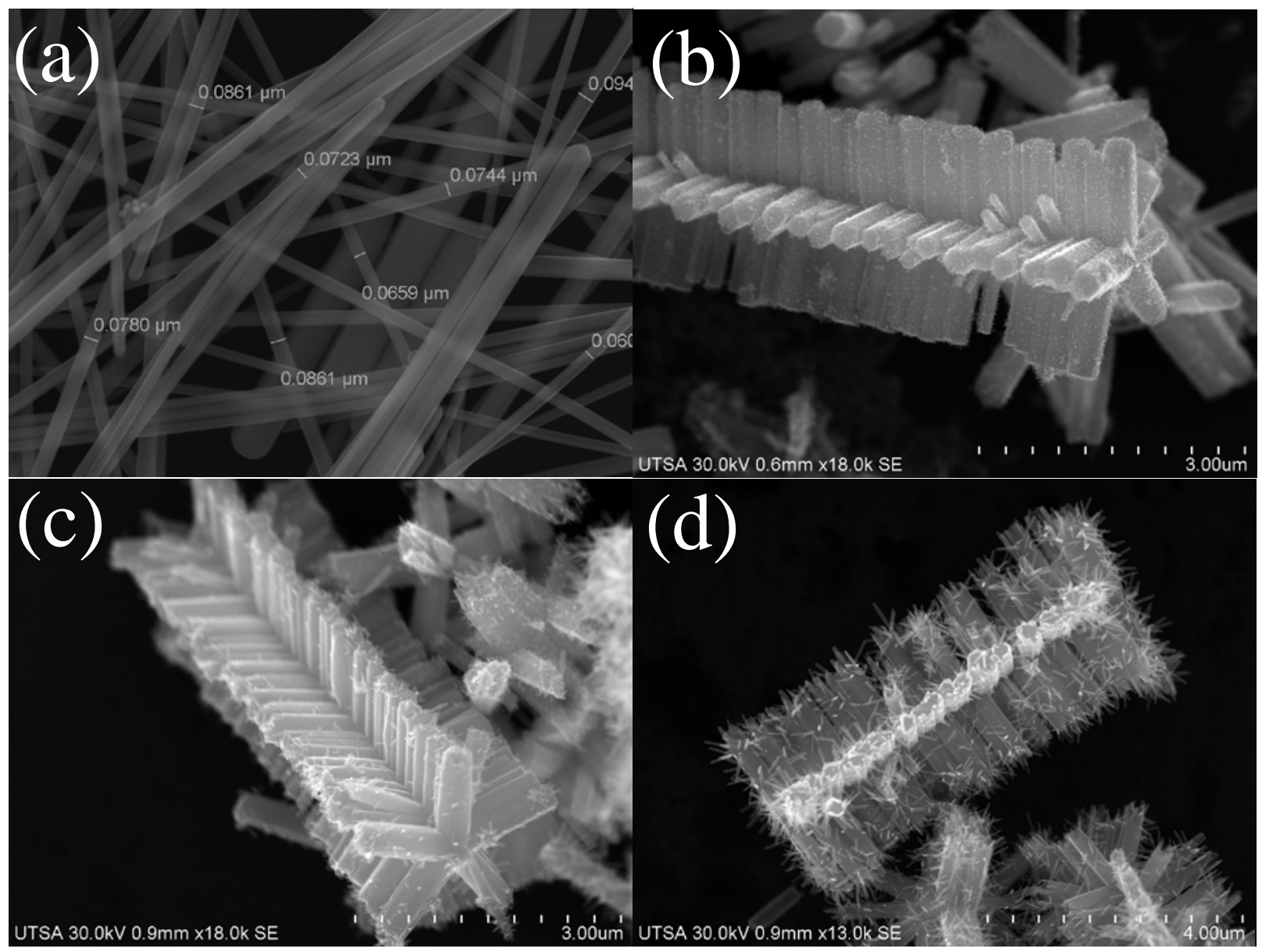


Figure 3. SEM images of (a) Ag nanowires, the diameter of the nanowire is shown in the Figure, it ranges from $70-90 \mathrm{~nm}$. (b) $\mathrm{Ag} / \mathrm{ZnO}$-cluster nanowiskers, first the $\mathrm{ZnO}$ nanowiskers starts to growth as nucleation points along $\mathrm{ZnO}$ nanorods (c) and (d) $\mathrm{Ag} / \mathrm{ZnO}$ after controlling the growth of clusters nanowiskers along $\mathrm{ZnO}$ nanorods by using $\mathrm{ZnO}$ nanoparticle seeds.

\section{Results and discussion}

\subsection{TCR Measurements}

The TCR, which represents the normalized change in resistance with respect to temperature, is calculated by using the following formula [1]

$$
\alpha=\frac{1}{R} \frac{d R}{d T},
$$

where $R$ represents the resistance and $T$ represents the temperature.

Resistance versus temperature measurements at room temperature resulted in a positive TCR around $1 \% \mathrm{~K}^{-1}$ for the silver nanowires films which presented a $16 \Omega$ resistance (Fig. 3(a)), the film made of $\mathrm{Ag} / \mathrm{ZnO}$ nanostructures showed a TCR of $-7.01 \% \mathrm{~K}^{-1}$, the negative TCR indicates the semiconductive nature of the film, the device showed an average resistance of $5 \mathrm{M} \Omega$ (Fig. 1(a)), while the device made from $\mathrm{Ag} / \mathrm{ZnO}$ nanostructures fabricated using zinc oxide nanoparticle seeds showed the highest TCR $\left(-11.8 \% \mathrm{~K}^{-1}\right)$ with a resistance of $2.2 \mathrm{M} \Omega$ (Fig. 3(d)). This increase in TCR can be explained by a greater amount of $\mathrm{ZnO}$ and an increase in carriers due to the silver nanowires, which can move rapidly with thermal drive leading to a higher TCR [8]. In table I we have summarized our results for the highest TCR values obtained for each sample. 
Table 1. Summary of the maximum TCR value for the three different analyzed samples.

\begin{tabular}{lll}
\hline Sample & Maximum TCR & Resistance \\
& $\left(\% \mathrm{~K}^{-1}\right)$ & $(\Omega)$ \\
\hline Ag nanowires & 1.04 & 16 \\
Ag/ZnO nanostructures & -7.07 & $5 \times 10^{6}$ \\
Ag/ZnO nanostructures (seeds) & -11.8 & $2.2 \times 10^{6}$ \\
& & \\
\hline
\end{tabular}

\subsection{Noise Measurements}

The voltage noise spectral density was measured in the frequency range of 1-100 kHz using a Hewlett-Packard HP3562A Dynamic Signal Analyzer, the noise of the system, and the total noise (system + noise of the device) were measured separately and then subtracted (in quadrature) to obtain the noise of the device [14].

Figure 4 shows the voltage noise measurements of all the bolometric samples at room temperature. From the figure it can be seen how the voltage noise of the devices at $1 \mathrm{kHz}$ is $90 \mathrm{nV} / \mathrm{Hz}^{-1 / 2}$ for the silver nanowires, $30 \mu \mathrm{V} / \mathrm{Hz}^{-1 / 2}$ for the $\mathrm{Ag} / \mathrm{ZnO}$ nanostructures and $90 \mu \mathrm{V} / \mathrm{Hz}^{-}$ 
1/2 for the Ag/ZnO nanostructures with seeds. It is worth noting how the voltage noise of the $\mathrm{Ag} / \mathrm{ZnO}$ nanostructures with seeds show a different behavior than the silver nanowires and regular $\mathrm{Ag} / \mathrm{ZnO}$ nanostructures, the $1 / f$ characteristics of the nanostructures does not decrease as fast as the other two devices, this could be due to different noise mechanisms associated to the metal-semiconductor barrier such as shot noise. A more detailed analysis of the sources of noise in theses types of nanostructured devices is needed in order to improve the $1 / \mathrm{f}$ noise characteristics of the bolometric device presented in this work. By being able to control the noise characteristics would further improve other figures of merit used to characterize bolometric devices such as Detectivity ( $\left.\mathrm{D}^{*}\right)$ for example.

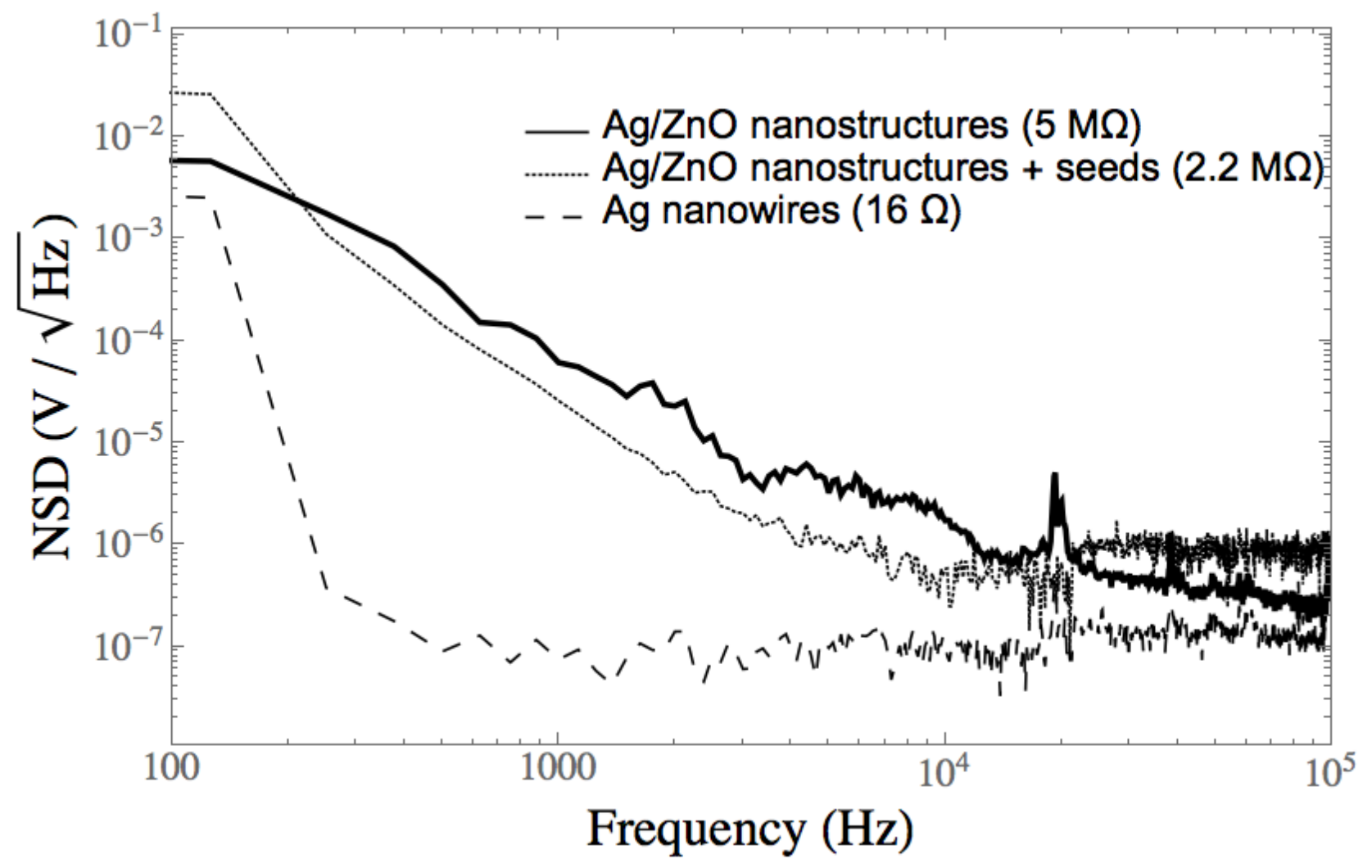

Figure 4. Noise spectral density of $\mathrm{Ag} / \mathrm{ZnO}$ nanostructured bolometers with different concentrations ratio. 


\subsection{Absorption Measurements}

Another important parameter for bolometer responsivity is the absorptivity. From Eq. (1) it can be seen that the responsivity is directly proportional to the absorption of the bolometric material, in some types of bolometers a special absorbing layer is used in order to increase the absorption of bolometric device, however it is desirable that the bolometric material has high absorption at the wavelengths of interest.

The infrared absorption spectra of the bolometric devices made from films of Ag nanowires, $\mathrm{Ag} / \mathrm{ZnO}$ nanostructures and $\mathrm{Ag} / \mathrm{ZnO}$ nanostructures with seeds, were taken with a Bruker IFS66v FTIR spectrometer with a near normal incidence $\left(7^{\circ}\right)$ geometry. The measurements were performed in a vacuum environment and at room temperature. For control purposes, absorption from the bolometric material films were performed using a bare Si wafer as a reference. Figure 5 shows the absorption measurements of the three bolometric films. From the figure it can be seen how the nanostructured material fabricated with seeds has a higher absorption than the regular $\mathrm{Ag} / \mathrm{ZnO}$ nanostructures, the average absorption for the nanostructured material with seeds is around $15 \%$ for the $5-15 \mu \mathrm{m}$ wavelength range. Due to the low absorption at the infrared wavelengths of interest an absoption layer would be needed, this will further increase the responsivity of the bolometric device. 


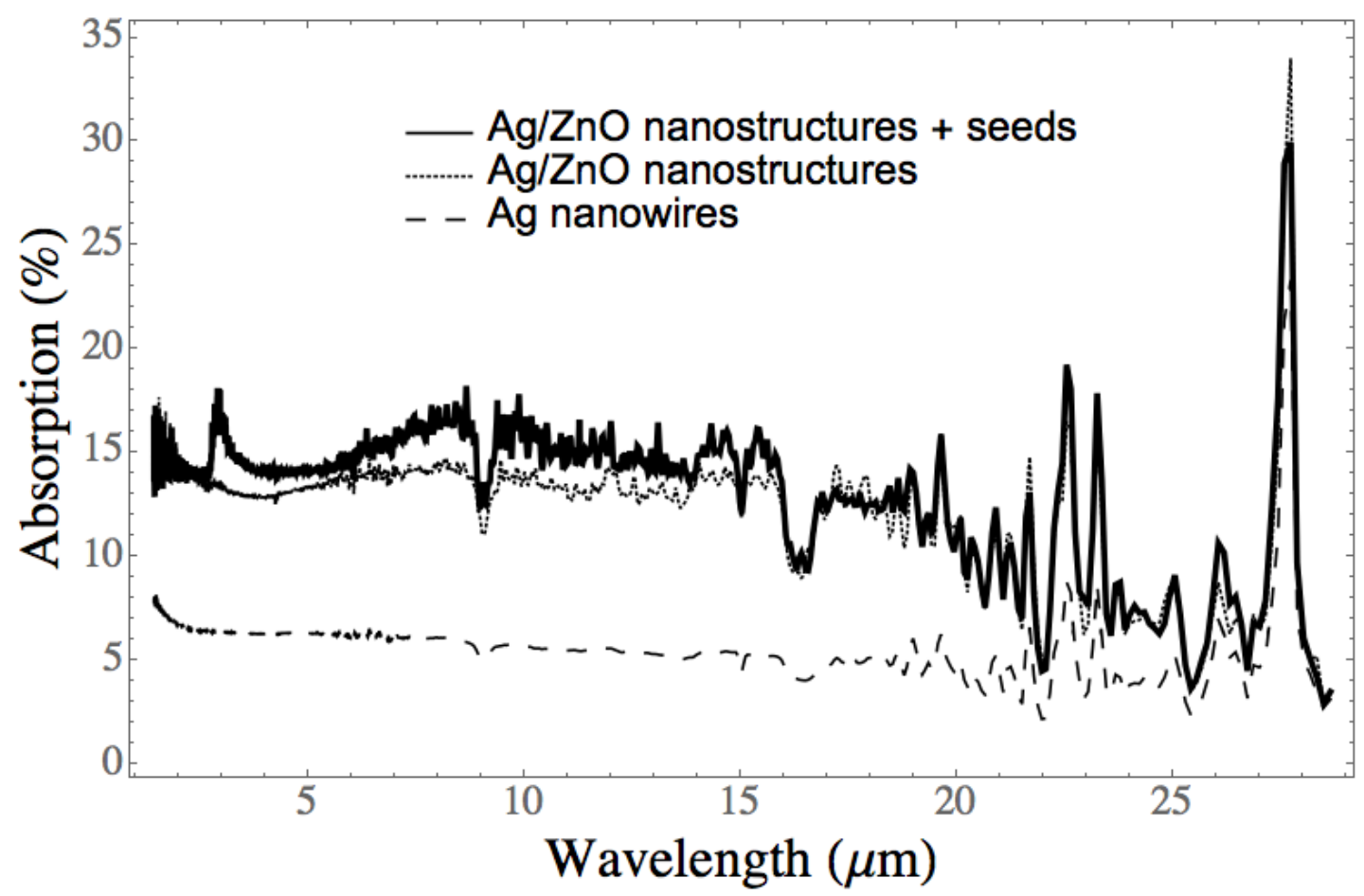

Figure 5. FTIR absorption spectra of $\mathrm{Ag} / \mathrm{ZnO}$ nanostructured bolometers with different concentrations ratio.

\section{Conclusions}

Measurements of thermo-electric parameters on self-assembled $\mathrm{Ag} / \mathrm{ZnO}$ nanostructures fabricated with seeds showed high room temperature TCR values up to $-11.8 \% \mathrm{~K}^{-1}$, with the added advantage that the TCR and conductivity of the material can be tuned with the Ag/ZnO concentration ratio. Also this self-assembled nanostructure showed good absorption at infrared wavelengths and noise values that can also be tuned by changing the $\mathrm{Ag} / \mathrm{ZnO}$ ratio. Results show that intermetallic-semiconductor nanostructures have a great potential for uncooled bolometric applications due to their electrical and optical properties that can be tailored depending on the concentration ratio used in their fabrication. This tuning can be used to increase the response of 
the device or to better match the device's electrical impedance to a read-out integrated for infrared imaging system applications.

\section{ACKNOWLEDGMENTS}

FJG would like to acknowledge support by project 32 of CEMIE-Solar from Fondo Sectorial CONACYT-Secretaría de Energía-Sustentabilidad Energética and by the National Laboratory program from CONACYT through the Terahertz Science and Technology National Lab (LANCYTT). AP acknowledges to PREI-DGAPA-UNAM for the economic support during the summer period. This work was also supported by the program "Cátedras CONACYT”. We also acknowledge the support by the NIH RCMI Nanotechnology and Human Health Core (G12MD007591), Department of Defense \#64756-RT-REP and the Welch Foundation grant award \#AX-1615.

\section{References}

[1] Dereniak, E.L.; Boreman, G.D. Infrared Detectors and Systems. 1996. Wiley Interscience.

[2] Watts, C. M.; Liu X.; Padilla,W. J. Metamaterial Electromagnetic Wave Absorbers. Adv. Mater. 2012; 24 (23): OP98-120, OP181.

[3] Jiang, Z. H.; Yun, S.; Toor, F.; Werner, D. H.; Mayer T. S. Conformal Dual-Band NearPerfectly Absorbing Mid-Infrared Metamaterial Coating. ACS Nano 2011, 5 (6), 4641-4647.

[4] Asano, T.; Chong, H.; Yi, Z.; Mingguo, L.; Campbell, J. C.; Madhukar, A. Design Consideration and Demonstration of Resonant-Cavity-Enhanced Quantum Dot Infrared Photodetectors in Mid-Infrared Wavelength Regime. IEEE J. Quantum Electron. 2010, 46, $1484-1491$. 
[5] Battal, E.; Bolat, S.; Tanrikulu, M. Y.; Okyay, A. K. and Akin, T. Atomic-Layer-Deposited Zinc Oxide as Tunable Uncooled Infrared Microbolometer Material. Phys. Status Solidi A 2014, 211, 2475-2482.

[6] Wang, B.; Lai, J.; Li, H.; Hu, H.; Chen, S. Nanostructured Vanadium Oxide Thin Film With High TCR at Room Temperature for Microbolometer, Infrared Phys. \& Techn. 2013, 57, 813.

[7] Choi, S.; Kim, B.J.; Lee, Y.W.; Yun, S.J.; Kim, H.T. Synthesis $\mathrm{Of}_{\mathrm{VO}_{2}}$ Nanowire and Observation of the Metal-Insulator Transition. Japanese Journal of Applied Physics 2008, 47, 3296-3298.

[8] Zhou, X. Ag-Doping Improving The Detection Sensitivity Of Bolometer Based On ZnO Thin Films. Vacuum 2015, 117, 47-49.

[9] Wang, S.; Yu, Y.; Zuo, Y.; Li, C.; Yang, J.; Lu, C. Synthesis and Photocatalysis of Hierarchical Heteroassemblies of ZnO Branched Nanorod Arrays on Ag Core Nanowires. Nanoscale 2012, 4(19), 5895-901.

[10] Sanchez, J. E.; Mendoza-Santoyo, F.; Cantu-Valle, J.; Velazquez-Salazar, J.; José-Yacaman, M.; González, F. J.; Díaz de Leon, R.; Ponce, A. Electric Radiation Mapping of Silver/Zinc Oxide Nanoantennas By Using Electron Holography. J. Appl. Phys. 2015, 117, 034306.

[11] Sun, Y.; Gates, B.; Mayers, B.; Xia, Y. Crystalline Silver Nanowires by Soft Solution Processing. Nano Letters, 2002, 2 (2), 165-168. 
[12] Sun, Y.; Yin, Y.; Mayers, B.T.; Herricks, T.; Xia, Y. Uniform Silver Nanowires Synthesis by Reducing AgNO3 with Ethylene Glycol in the Presence of Seeds and Poly(Vinyl Pyrrolidone). Chem. Mater., 2002, 14 (11), 4736-4745.

[13] Santiago, U.; Velázquez-Salazar, J. J.; Sanchez, J. E.; Ruiz-Zepeda, F.; Ortega, J. E.; ReyesGasga, J.; Bazán-Díaz, L.; Betancourt, I.; Rauch, E. F.; Veron, M.; Ponce A.; José-Yacamán M. A Stable Multiply Twinned Decahedral Gold Nanoparticle with a Barrel-Like Shape. Surface Science 2016, 644, 80-85.

[14] González F. J. Noise Measurements on Optical Detectors. Revista Mexicana de Física 2006, 52 (6), 550-554. 